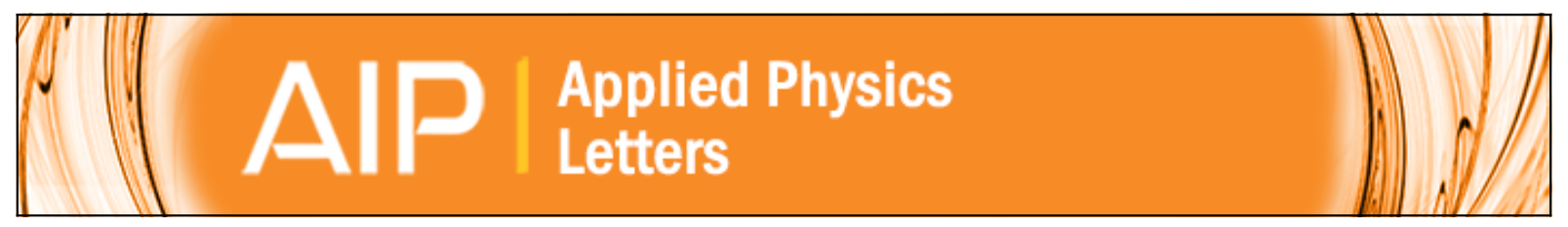

Reliable low-power control of ultrafast vortex-core switching with the selectivity in an array of vortex states by in-plane circular-rotational magnetic fields and spin-polarized currents

Sang-Koog Kim, Ki-Suk Lee, Young-Sang Yu, and Youn-Seok Choi

Citation: Applied Physics Letters 92, 022509 (2008); doi: 10.1063/1.2807274

View online: http://dx.doi.org/10.1063/1.2807274

View Table of Contents: http://scitation.aip.org/content/aip/journal/apl/92/2?ver=pdfcov

Published by the AIP Publishing

Articles you may be interested in

Current- and field-driven magnetic antivortices for nonvolatile data storage

Appl. Phys. Lett. 94, 062504 (2009); 10.1063/1.3072342

Understanding eigenfrequency shifts observed in vortex gyrotropic motions in a magnetic nanodot driven by spinpolarized out-of-plane dc current

Appl. Phys. Lett. 93, 182508 (2008); 10.1063/1.3012380

Spin transfer switching of closely arranged multiple pillars with current-perpendicular-to-plane spin valves

J. Appl. Phys. 103, 07 A713 (2008); 10.1063/1.2838473

Spin-current pulse induced switching of vortex chirality in permalloy/Cu/Co nanopillars

Appl. Phys. Lett. 91, 022501 (2007); 10.1063/1.2756109

MRAM array with coupled soft-adjacent magnetic layer

J. Appl. Phys. 97, 10P506 (2005); 10.1063/1.1855732

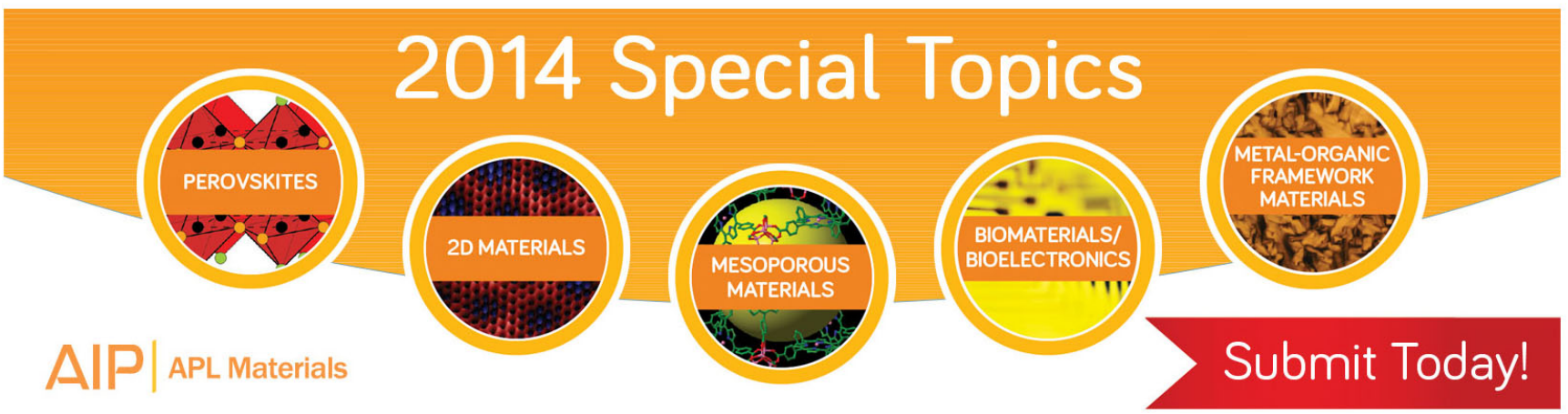




\title{
Reliable low-power control of ultrafast vortex-core switching with the selectivity in an array of vortex states by in-plane circular-rotational magnetic fields and spin-polarized currents
}

\author{
Sang-Koog Kim, ${ }^{\text {a) }}$ Ki-Suk Lee, Young-Sang Yu, and Youn-Seok Choi \\ Research Center for Spin Dynamics and Spin-Wave Devices, and Nanospintronics Laboratory, \\ Department of Materials Science and Engineering, College of Engineering, Seoul National University, \\ Seoul 151-744, Republic of Korea
}

(Received 24 September 2007; accepted 19 October 2007; published online 16 January 2008)

\begin{abstract}
The authors investigated the technological utility of counterclockwise $(\mathrm{CCW})$ and clockwise $(\mathrm{CW})$ circular-rotating fields $\left(\mathbf{H}_{\mathrm{CCW}}\right.$ and $\left.\mathbf{H}_{\mathrm{CW}}\right)$ and spin-polarized currents with an angular frequency $\omega_{\mathbf{H}}$ close to the vortex eigenfrequency $\omega_{D}$, for the reliable, low-power, and selective switching of the bistate magnetization $(\mathbf{M})$ orientations of a vortex core (VC) in an array of soft magnetic nanoelements. $\mathrm{CCW}$ and $\mathrm{CW}$ circular gyrotropic motions in response to $\mathbf{H}_{\mathrm{CCW}}$ and $\mathbf{H}_{\mathrm{CW}}$, respectively, show remarkably contrasting resonant behaviors, (i.e., extremely large-amplitude resonance versus small-amplitude nonresonance), depending on the $\mathbf{M}$ orientation of a given VC. Owing to this asymmetric resonance characteristics, the $\mathbf{H}_{\mathrm{CCW}}\left(\mathbf{H}_{\mathrm{CW}}\right)$ with $\omega_{\mathbf{H}} \sim \omega_{D}$ can be used to effectively switch only the up (down) core to its downward (upward) $\mathbf{M}$ orientation, selectively, by sufficiently low field $(\sim 10 \mathrm{Oe})$ and current density $\left(\sim 10^{7} \mathrm{~A} / \mathrm{cm}^{2}\right)$. This work provides a reliable, low power, effective means of information storage, information recording, and information readout in vortex-based random access memory, simply called VRAM. (C) 2008 American Institute of Physics. [DOI: 10.1063/1.2807274]
\end{abstract}

A magnetic vortex (MV) is a ground state of magnetization (M) configuration in soft magnetic elements of submicron lateral size or smaller, ${ }^{1}$ as well as in continuous magnetic thin films. ${ }^{2}$ The micromagnetic structure of the MV consists of out-of-plane Ms at the vortex core (VC) and inplane curling Ms around it. ${ }^{1-3}$ The VC is of either upward or downward $\mathbf{M}$ orientation, and the remaining in-plane $\mathbf{M s}$ around the $\mathrm{VC}$ are also of either clockwise $(\mathrm{CW})$ or counterclockwise $(\mathrm{CCW})$ rotation. These $\mathbf{M}$ configurations are stable down to sufficiently small nanoelements of lateral size of $>100 \mathrm{~nm}$ and thickness of $>10 \mathrm{~nm}$ (Ref. 4). Owing to the bistates of the $\mathrm{VC}$ orientation, technological interest in the practical applicability of the MVs to future informationstorage devices ${ }^{5}$ such as vortex-based random access memory, simply called VRAM, has rapidly grown. In order to use the vortex state as a memory unit, however, lowpower, reliable control of VC switching between its upward and downward $\mathbf{M}$ orientations with the selectivity of a single vortex in an array of them is necessary. Very recently, ultrafast VC switching has been practically realized in experiments, by applying, with sufficiently low power, either externally applied linearly oscillating magnetic field pulse ${ }^{6}$ or a spin-polarized currents ${ }^{7}$ in the film plane. Much and increasing attention thus has been paid to vortex dynamics ${ }^{8,9}$ including VC reversal (switching), ${ }^{10-13}$ gyrotropic motions, ${ }^{14,15}$ and their relations ${ }^{16,17}$ in confined magnetic elements, from both scientific and technological points of view.

In this letter, we report, as a further step, a promisingly effective, extremely low-power means of manipulating VC switching and vortex motions by the use of $\mathrm{CCW}$ and $\mathrm{CW}$ circular-rotational fields ( $\mathbf{H}_{\mathrm{CCW}}$ and $\mathbf{H}_{\mathrm{CW}}$ ) and spin-polarized currents $\left(\mathbf{I}_{\mathrm{CCW}}\right.$ and $\left.\mathbf{I}_{\mathrm{CW}}\right)$ in the film plane with their angular

\footnotetext{
${ }^{\text {a) }}$ Author to whom correspondence should be addressed. Electronic mail:
} sangkoog@snu.ac.kr. frequencies $\omega_{\mathbf{H}, \mathbf{I}}$ close to the angular eigenfrequecy $\omega_{D}$ of the vortex translation mode. ${ }^{18}$ These circular fields and currents are more effective than linearly alternating fields and currents to switch a VC chosen among an array of vortex states, as will be demonstrated.

In the present study, we employed mircomagnetic simulations of vortex dynamics driven by externally applied fields $\mathbf{H}_{i}$ and currents $\mathbf{I}_{i}$ using the OOMMF (Ref. 19) and LLG (Ref. 20) codes, respectively, where the subscript $i$ denotes corresponding $\mathrm{CCW}$ and $\mathrm{CW}$ circular-rotational, and linear oscillating fields or currents, respectively. Both codes, based on the Landau-Lifshitz-Gilbert (LLG) equation of motion, provide the same results for the $\mathbf{M}$ dynamics driven by applied magnetic fields. For current-driven $\mathbf{M}$ dynamics, we used the LLG code (for details on the spin torque term and parameters, see Ref. 16, and references therein). For the $\mathbf{I}_{\mathrm{CCW}, \mathrm{CW}}$ applications, we employed two conducting electrodes orthogonally crossing each other on each vortex, and which are practically applicable to the applications of $\mathbf{I}_{\mathrm{CCW}, \mathrm{CW}}$ or $\mathbf{H}_{\mathrm{CCW}, \mathrm{CW}}$ in potential random-access-memory devices based on an array of magnetic nanoelements where single vortex states are stable. As a model system, we chose a Permalloy (Py) nanodisk of $2 R=600 \mathrm{~nm}$ diameter and $L=20 \mathrm{~nm}$ thickness. In the Py disk, a single MV of up- or down-core orientation and with $\mathrm{CCW}$ in-plane $\mathbf{M}$ rotation was chosen as an initial ground state at equilibrium under no external perturbation, as shown in the left column of Fig. 1. In the given model system, the vortex eigenfrequency $\nu_{D}=\omega_{D} / 2 \pi$ with a damping constant $D=0.01$ (Ref. 21) was estimated to be $300 \mathrm{MHz}$, through the relaxation from a shift of the initial position of a VC by a static field of 160 Oe applied in the $+y$ direction (middle column of Fig. 1). Each VC follows a logarithmic spiral orbital motion ${ }^{22}$ with the CCW rotation for the up-core orientation, but with the $\mathrm{CW}$ rotation for the down-core orientation under no external driving force. In addition, under a 


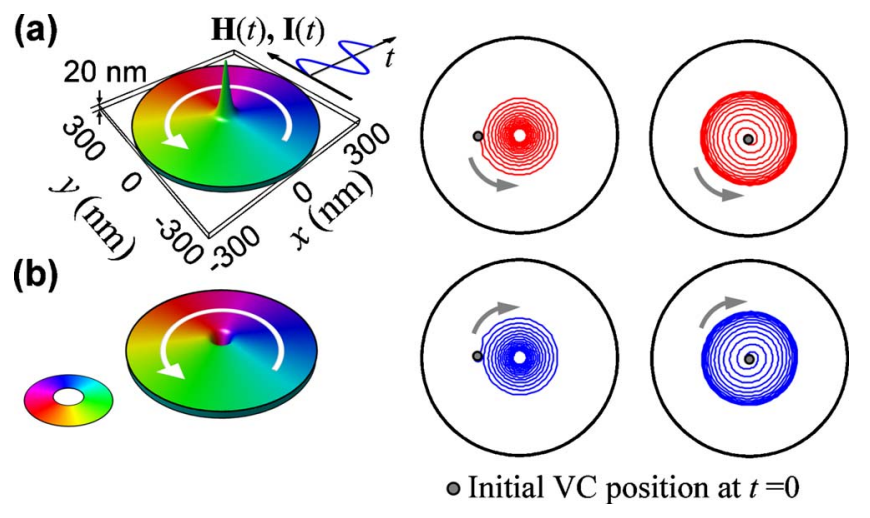

FIG. 1. (Color online) Left column: Geometry and dimensions of the model Py nanodisk, and $\mathbf{M}$ distributions of vortex states with (a) up- and (b) down-core orientations, with the $\mathrm{CCW}$ rotation of in-plane Ms around the VC. The color and height display the local Ms and their out-of-plane components, respectively. The middle and right columns display the VC trajectories of the vortex gyrotropic motions during relaxation $\left(H_{0}=0\right)$ from a shift of each VC from its initial center position, and during the vortex motion from its initial center position under $\mathbf{H}_{\text {lin }}(t)$ with $H_{0}=10$ Oe and $\nu_{\mathbf{H}}$ at $\nu_{D}=300 \mathrm{MHz}$, respectively. The red and blue colors denote the VC trajectories of the up- and down-core orientations, respectively.

linearly oscillating magnetic field in the $y$ axis $\left[\mathbf{H}_{\text {lin }}\right.$ $\left.=H_{0} \sin \left(2 \pi \nu_{\mathbf{H}} t\right) \hat{\mathbf{y}}\right]$, with $H_{0}=10$ Oe and $\nu_{\mathbf{H}}$ at $\nu_{D}$ $=300 \mathrm{MHz}$, for example, the $\mathrm{VC}$ shows similar $\mathrm{CCW}(\mathrm{CW})$ spiral motion in the initial transient state for the up (down) core while moving from the initial center position outward, but finally attains a steady-state circular motion of a finitesize orbital radius, as seen in the right column of Fig. 1.

It is quite interesting that the rotation sense of the $\mathrm{VC}$ gyrotropic motions is determined only by the $\mathbf{M}$ orientation (polarity) of the VC (Ref. 18) in both cases where no driving force and a linearly oscillating field are applied. In fact, the $\mathbf{H}_{\text {lin }}\left(\right.$ or $\mathbf{I}_{\text {lin }}$ ) for the $\mathrm{VC}$ resonant motions shown in Fig. 1 consists of (i.e., is decomposed into) $\mathrm{CCW}$ and $\mathrm{CW}$ circular components rotating with $\omega_{\mathbf{H , I}}$ in the film plane with their equal oscillating amplitude, as denoted by $\mathbf{H}_{\mathrm{CCW}}(t)$ $=H_{0} \cos \left(\omega_{\mathbf{H}} t\right) \hat{\mathbf{x}}+H_{0} \sin \left(\omega_{\mathbf{H}} t\right) \hat{\mathbf{y}}$ and $\mathbf{H}_{\mathrm{CW}}(t)=-H_{0} \cos \left(\omega_{\mathbf{H}} t\right) \hat{\mathbf{x}}$ $+H_{0} \sin \left(\omega_{\mathbf{H}} t\right) \hat{\mathbf{y}}$ (see Fig. 2). Therefore, in order to understand the observed resonant circular motions and true eigenmodes ${ }^{23}$ of VCs under an arbitrary polarized oscillating field, as well as the $\mathrm{VC}$ switching driven by $\mathbf{H}_{\mathrm{CCW}, \mathrm{CW}}$ (or $\mathbf{I}_{\mathrm{CCW}, \mathrm{CW}}$ ), it is necessary to find the elementary eigenmodes of the VC motions in circular nanodisks by applying $\mathbf{H}_{\mathrm{CCW}, \mathrm{CW}}$ and $\mathbf{I}_{\mathrm{CCW}, \mathrm{Cw}}$. Such orthogonal circular fields or currents can, in principle, be achieved practically by applying linearly oscillating fields or currents through the use of two electrodes orthogonally crossing each other, with the phase difference of either $+90^{\circ}$ or $-90^{\circ}$ between the oscillating currents flowing along the different electrodes, for example, $H_{x}\left(I_{x}\right)$ and $H_{y}\left(I_{y}\right)$ as shown in Figs. 2(b) and 2(c).

By the uses of $\mathbf{H}_{\mathrm{CCW}}$ and $\mathbf{H}_{\mathrm{CW}}$ as well as $\mathbf{H}_{\text {lin }}$, the switching and gyrotropic motions of the upward VC have been simulated according to $\omega_{\mathbf{H}}=2 \pi \nu_{\mathbf{H}}$, as seen in Fig. 3(a), where the orbital trajectories $T_{\text {orb }}(\mathbf{X})$ of the VC position $\mathbf{X}$ are plotted during a time interval of $t=0-32$ ns upon applying the given fields. Here, we note that the dimensions of the model Py disk and a field strength of $H_{0}=10$ Oe were chosen to achieve the VC switching only by $\mathbf{H}_{\mathrm{CCW}}$ at $\omega_{\mathbf{H}} \sim \omega_{D}$, not by $\mathbf{H}_{\mathrm{CW}}$ and $\mathbf{H}_{\text {lin }}$ for the up-core orientation. ${ }^{24}$ Figure $3(\mathrm{~b})$ shows the temporal evolution of the $\mathrm{VC}$ switching at the indicated times, representing the underlying mechanism of
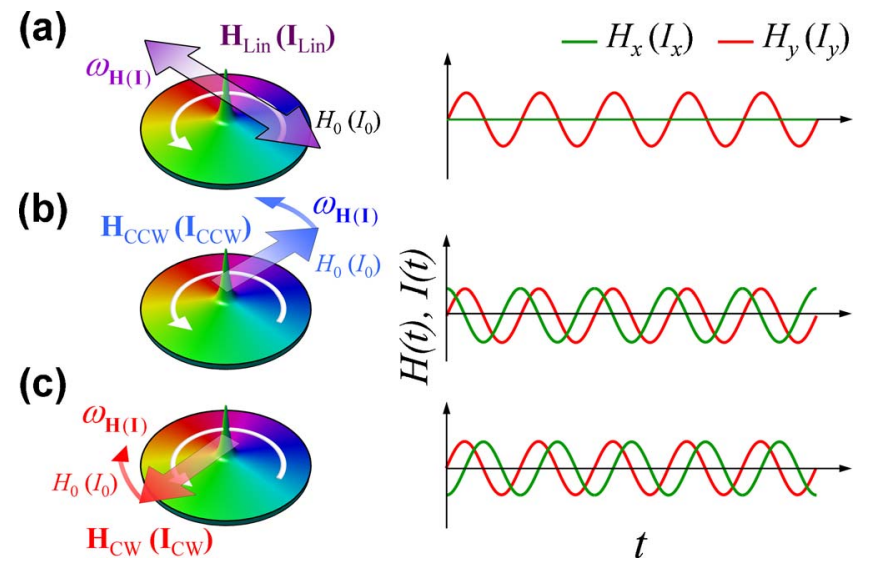

FIG. 2. (Color online) Illustrations of $\mathbf{H}_{\text {lin }}\left(\mathbf{I}_{\text {lin }}\right)$ in (a), of $\mathbf{H}_{\mathrm{CCW}}\left(\mathbf{I}_{\mathrm{CCW}}\right)$ in (b), and of $\mathbf{H}_{\mathrm{CW}}\left(\mathbf{I}_{\mathrm{CW}}\right)$ in (c), rotating with $\omega_{\mathbf{H}(\mathbf{I})}$ in the film plane. The right column shows the time variation of the magnitude of the field or current components along the $x$ and $y$ axes. The sum of $\mathbf{H}_{\mathrm{CCW}}\left(\mathbf{I}_{\mathrm{CCW}}\right)$ and $\mathbf{H}_{\mathrm{CW}}\left(\mathbf{I}_{\mathrm{CW}}\right)$ with equal $\omega_{\mathbf{H}(\mathbf{I})}$ and $H_{0}\left(j_{0}\right)$ leads to $\mathbf{H}_{\text {lin }}\left(\mathbf{I}_{\text {lin }}\right)$ with the same $\omega_{\mathbf{H}(\mathbf{I})}$. The superposition of the orthogonal circular fields results in a linear oscillating field, such that $\left(\mathbf{H}_{\mathrm{CCW}}+\mathbf{H}_{\mathrm{CW}}\right) / 2=H_{0} \sin \left(\omega_{\mathbf{H}} t\right) \hat{\mathbf{y}}=\mathbf{H}_{\text {lin }}$. According to the phase difference between $\mathbf{H}_{\mathrm{CCW}}\left(\mathbf{I}_{\mathrm{CCW}}\right)$ and $\mathbf{H}_{\mathrm{CW}}\left(\mathbf{I}_{\mathrm{CW}}\right)$, the direction of the linear field is determined.

how the VC reversal takes place in tens of picosecond scale or longer. ${ }^{6,9-13,17}$ For the down-core orientation, only the $\mathbf{H}_{\mathrm{CW}}$ with $H_{0}=10$ Oe at $\omega_{\mathbf{H}}=\omega_{D}$ allows the VC to switch to its reversed $\mathbf{M}$ orientation.

To understand why the $\mathrm{VC}$ switching is responding differently to $\mathbf{H}_{\mathrm{lin}}, \mathbf{H}_{\mathrm{CCW}}$, and $\mathbf{H}_{\mathrm{CW}}$, we should know the elementary eigenmodes of the vortex motion in circular nanodisks. As seen in Fig. 3(a), the rotation sense of the VC motion in response to $\mathbf{H}_{\mathrm{CCW}}$ is always CCW, but is $\mathrm{CW}$ in response to $\mathbf{H}_{\mathrm{CW}}$. However, the orbital radii for the CCW motion are extremely large compared with those for the CW motion in a wide range of $\omega_{\mathbf{H}}$ across $\omega_{D}$. When $\omega_{\mathbf{H}}$ reaches $\omega_{D}$, the orbital radius for the CCW rotation markedly increases even by a sufficiently small field strength of $H_{0}$ $=10 \mathrm{Oe}$, owing to the resonance characteristics. By contrast,

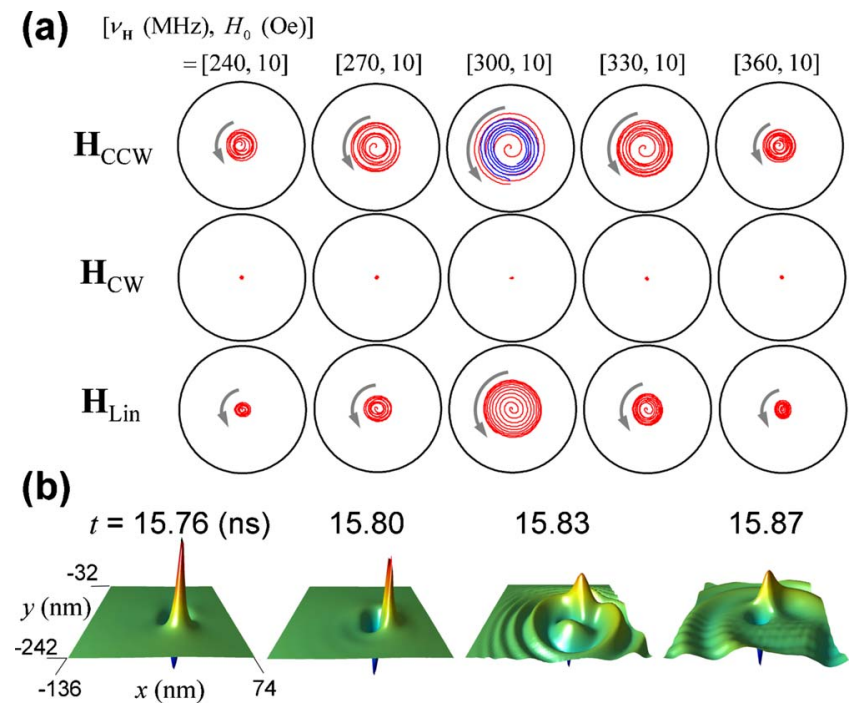

FIG. 3. (Color online) (a) VC trajectories of gyrotropic motions of a VC driven by $\mathbf{H}_{\text {lin }}, \mathbf{H}_{\mathrm{CCW}}$, and $\mathbf{H}_{\mathrm{CW}}$ for different values of $\nu_{\mathbf{H}}$ as noted. The red and blue colors of the trajectories indicate the motions of the initial up-core orientation and of the reversed VC after the VC switching, respectively. (b) Perspective snanshot images of the temporal evolution of VC reversals at the indicated times. 
(a) $\left[v_{\mathrm{r}}(\mathrm{MHz}), j_{0}\left(\times 10^{7} \mathrm{~A} / \mathrm{cm}^{2}\right)\right]$

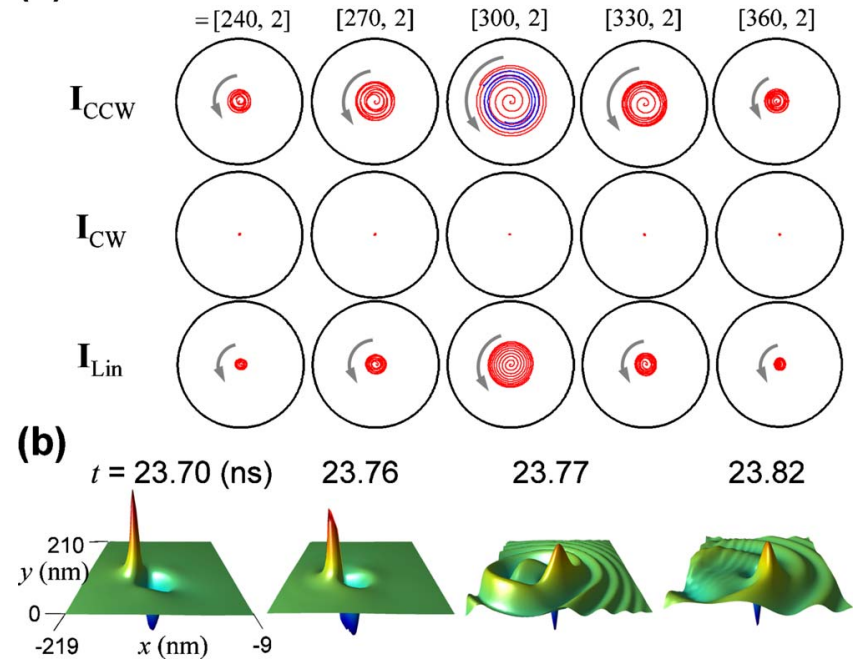

FIG. 4. (Color online) All of the data are presented in the same way as those in Fig. 3, except for the application of $\mathbf{I}_{\text {lin }}, \mathbf{I}_{\mathrm{CCW}}$, and $\mathbf{I}_{\mathrm{CW}}$ with a current density of $j_{0}=2 \times 10^{7} \mathrm{~A} / \mathrm{cm}^{2}$.

the $\mathrm{VC}$ motion driven by the $\mathbf{H}_{\mathrm{CW}}$ shows nonresonance with extremely small-amplitude orbits, as seen in the second row of Fig. 3(a). It is evident that the CCW and CW circular eigenmodes exhibit resonance and nonresonance characteristics, respectively, for the up-core orientation, and vice versa for the down-core orientation. This asymmetric resonance surely affects the reversal (switching) of a given VC depending on its $\mathbf{M}$ orientation. It is known from our earlier work $^{16,17}$ that the velocity of $\mathrm{VC}$ motion is required to reach its critical value of $v_{\text {cri }} \sim 350 \pm 20 \mathrm{~m} / \mathrm{s}$ in order to effect VC switching. Accordingly, the $\mathbf{H}_{\mathrm{CCW}}$ with $H_{0} \geqslant 10$ Oe at $\omega_{\mathbf{H}}$ $\sim \omega_{D}$ can selectively switch only the up core, but the $\mathbf{H}_{\mathrm{CW}}$ can switch only the down core because the $\mathbf{H}_{\mathrm{CCW}}\left(\mathbf{H}_{\mathrm{CW}}\right)$ steers only the up (down) core to reach the value of $v_{\text {cri }}$ with $H_{0} \geqslant 10$ Oe at $\omega_{\mathbf{H}} \sim \omega_{D}$. However, for the cases of $\omega_{\mathbf{H}}$ being far away from $\omega_{D}$, the strength of $H_{0}=10$ Oe is not sufficient to reach the $v_{\text {cri }}$ value, but leads to a steady-state circular motion. ${ }^{15,16}$

We also conducted micromagnetic simulations for such VC switching driven by spin-polarized currents with a sufficiently low current density of $j_{0}=2 \times 10^{7} \mathrm{~A} / \mathrm{cm}^{2}$. Very similar results were obtained, as can be seen in Fig. 4. Note that a linearly oscillating current larger than its critical density can also switch a VC at $\omega_{\mathbf{I}} \sim \omega_{D}$, as reported in Ref. 16. In this letter, we only chose the dimensions of the model geometry as well as the strength of external driving forces in order to achieve the $\mathrm{VC}$ switching by $\mathbf{H}_{\mathrm{CCW}}\left(\mathbf{I}_{\mathrm{CCW}}\right)$ or $\mathbf{H}_{\mathrm{CW}}\left(\mathbf{I}_{\mathrm{CW}}\right)$ not by $\mathbf{H}_{\text {lin }}\left(\mathbf{I}_{\text {lin }}\right)$. In an array of vortex states, we can switch a single vortex chosen to be recorded by flowing linearly oscillating currents along two electrodes crossing on only the selected vortex, with $+90^{\circ}$ or $-90^{\circ}$ phase difference, depending on the $\mathbf{M}$ orientation of the chosen vortex. Also, for detecting the $\mathrm{VC}$ orientations, we can utilize $\mathbf{H}_{\mathrm{CCW}}\left(\mathbf{I}_{\mathrm{CCW}}\right)$ or $\mathbf{H}_{\mathrm{CW}}\left(\mathbf{I}_{\mathrm{CW}}\right)$ because the given $\mathrm{VC}$ remarkably responds only to either $\mathbf{H}_{\mathrm{CCW}}\left(\mathbf{I}_{\mathrm{CCW}}\right)$ or $\mathbf{H}_{\mathrm{CW}}\left(\mathbf{I}_{\mathrm{CW}}\right)$, depending on its $\mathbf{M}$ orientation.

In conclusion, the $\mathrm{CCW}$ and $\mathrm{CW}$ rotating motions of VCs in response to pure $\mathbf{H}_{\mathrm{CCW}}$ and $\mathbf{H}_{\mathrm{CW}}$ (or $\mathbf{I}_{\mathrm{CCW}}$ and $\mathbf{I}_{\mathrm{CW}}$ ), respectively, in circular nanodisks exhibit a highly asymmet- ric resonance effect, that is, resonance for the $\mathrm{CCW}$ motion vs non-resonance for the $\mathrm{CW}$ motion, for the up-core, but opposite effect for the down-core. Thus, this asymmetric resonance behavior between the CCW and CW circular motions can be used effectively not only for reliable, low-power selective switching of bistate $\mathrm{VC}$ orientations by choosing either $\mathbf{H}_{\mathrm{CCW}}\left(\mathbf{I}_{\mathrm{CCW}}\right)$ or $\mathbf{H}_{\mathrm{CW}}\left(\mathbf{I}_{\mathrm{CW}}\right)$, but also for detection of the orientation of a given $\mathrm{VC}$, because the up (down)-core orientation responds up to its reversal only to $\mathbf{H}_{\mathrm{CCW}}\left(\mathbf{H}_{\mathrm{CW}}\right)$ when $\omega_{\mathbf{H}}$ reaches $\omega_{D}$ through the resonance effect. This work offers a principal further step toward practical applications of vortex states for ultrafast information-storage, -recording and -readout in vortex-based random access memory (VRAM).

This work was supported by Creative Research Initiatives (ReC-SDSW) of MOST/KOSEF.

${ }^{1}$ T. Shinjo, T. Okuno, R. Hassdorf, K. Shigeto, and T. Ono, Science 289, 930 (2000); A. Wachowiak, J. Wiebe, M. Bode, O. Pietzsch, M. Morgenstern, and R. Wiesendanger, ibid. 298, 577 (2002).

${ }^{2}$ S.-K. Kim, J. B. Kortright, and S.-C. Shin, Appl. Phys. Lett. 78, 2742 (2001); S.-K. Kim, K.-S. Lee, B.-W. Kang, K.-J. Lee, and J. B. Kortright, ibid. 86, 052504 (2005).

${ }^{3}$ A. Hubert and R. Schafer, Magnetic Domains (Springer, Berlin, 1998).

${ }^{4}$ K. L. Metlov and K. Y. Guslienko, J. Magn. Magn. Mater. 242-245, 1015 (2002).

${ }^{5}$ R. P. Cowburn, Nat. Mater. 6, 255 (2007); J. Thomas, Nat. Nanotechnol. 2, 206 (2007).

${ }^{6}$ B. Van Waeyenberge, A. Puzic, H. Stoll, K. W. Chou, T. Tyliszczak, R. Hertel, M. Fähnle, H. Brückl, K. Rott, G. Reiss, I. Neudecker, D. Weiss, C. H. Back, and G. Schütz, Nature (London) 444, 461 (2006).

${ }^{7}$ K. Yamada, S. Kasai, Y. Nakatani, K. Kobayashi, H. Kohno, A. Thiaville, and T. Ono, Nat. Mater. 6, 269 (2007).

${ }^{8}$ K.-S. Lee, B.-W. Kang, Y.-S. Yu, and S.-K. Kim, Appl. Phys. Lett. 85, 1568 (2004).

${ }^{9}$ K.-S. Lee, S. Choi, and S.-K. Kim, Appl. Phys. Lett. 87, 192502 (2005).

${ }^{10}$ R. Hertel, S. Gliga, M. Fähnle, and C. M. Schneider, Phys. Rev. Lett. 98, 117201 (2007).

${ }^{11}$ K.-S. Lee, K. Y. Guslienko, J.-Y. Lee, and S.-K. Kim, Phys. Rev. B 76, 174410 (2007).

${ }^{12}$ S. Choi, K.-S. Lee, K. Y. Guslienko, and S.-K. Kim, Phys. Rev. Lett. 98, 087205 (2007).

${ }^{13}$ V. P. Kravchuk, D. D. Sheka, Y. Gaididei, and F. G. Mertens, J. Appl. Phys. 102, 043908 (2007).

${ }^{14}$ S. Kasai, Y. Nakatani, K. Kobayshi, H. Kohno, and T. Ono, Phys. Rev. Lett. 97, 107204 (2006); J. Magn. Magn. Mater. 310, 2351 (2007).

${ }^{15}$ K.-S. Lee and S.-K. Kim, Appl. Phys. Lett. 91, 132511 (2007).

${ }^{16}$ S.-K. Kim, Y.-S. Choi, K.-S. Lee, K. Y. Guslienko, and D.-E. Jeong, Appl. Phys. Lett. 91, 082506 (2007).

${ }^{17}$ K. Y. Guslienko, K.-S. Lee, and S.-K. Kim, e-print arXiv:cond-mat/ 0708.1359 .

${ }^{18}$ K. Y. Guslienko, B. A. Ivanov, V. Novosad, Y. Otani, H. Shima, and K. Fukamichi, J. Appl. Phys. 91, 8037 (2002).

${ }^{19} \mathrm{See}$ http://math.nist.gov/oommf.

${ }^{20} \mathrm{See}$ http://llgmicro.home.mindspring.com.

${ }^{21}$ For the case of a free oscillation of a VC with no damping $(D=0)$, the VC shows a circular motion around its equilibrium position with the eigenfrequency $\omega_{0}$, as defined analytically in Ref. 18 . For the case with the damping $(D \neq 0)$, the VC shows a logarithmic spiral motion as shown in Fig. 1 with the eigenfrequency $\omega_{D}$ that varies slightly with $D$. From the numerical solution of analytical equation of motion (Ref. 15) the $\omega_{D}$ value for $D=0.01$ is estimated to be $\omega_{D}=0.9993 \omega_{0}$ (i.e., $\omega_{D} \sim \omega_{0}$ ).

${ }^{22}$ The orbital radius of the VC gyrotropic motion decreases exponentially with time.

${ }^{23}$ K.-S. Lee, S.-K. Kim, D.-E. Jung, Y.-S. Yu, and Y.-S. Choi, arXiv:condmat/0712.0758.

${ }^{24}$ In Ref. 13, such asymmetric VC switching behavior in response to $\mathbf{H}_{\mathrm{CCW}}$ and $\mathbf{H}_{\mathrm{CW}}$ was reported only in a high frequency range $(\sim 10 \mathrm{GHz})$ above $\omega_{D}$ 